\title{
Fatigue in rheumatology: what can we target for treatment?
}

\begin{abstract}
"...treatment should comprise a best-fit approach ... this will be achieved through the modification and application of additional management programs, which should comprise multidisciplinary nonpharmacological interventions, to be initiated alongside pharmaceutical disease management strategies."
\end{abstract}

Keywords: fatigue $\bullet$ rheumatology $\bullet$ treatment

Fatigue is an irrefutably important patient priority across the rheumatic diseases. It is pervasive: as many as $80 \%$ of rheumatoid arthritis patients report clinically relevant levels, with similar if not higher rates observed among other forms of rheumatic disease [1,2] . Fatigue's impact permeates almost all spheres of life. The symptom, which has an unrelenting but unpredictable nature, is closely associated with disability and is rated as a central barrier to maintaining employment [3,4]. Yet, fatigue manifests as cognitive as well as physical fatigue, which means its impact spans personal roles, daily activities, emotions and relationships.

Little is known about how best to target the symptom for treatment. For many years research in the field has targeted the development of validated measures. However, while of course vital, we are now left with a plethora of nonstandardized tools and an absence of consensus regarding how they may be optimally applied. The subsequent confusion among clinicians and trialists will inevitably lead to barriers for the robust review and comparison of existing and future studies. In the meantime the significant burden and impact of the symptom prevails. Patients, who often consider the symptom to be of little interest to physicians, largely engage in self-management strategies such as pacing, resting, distraction and prioritizing [2]; however, the efficacy of such approaches has yet to be tested and may even be deleterious in their effect as these strategies could be associated with increased social withdrawal and isolation. The lack of recommended treatment for this symptom demands immediate attention.
To best treat fatigue, it is necessary to understand its etiology. Accordingly a growing body of promising work has begun to elucidate the biological determinants of fatigue. Sophisticated neuroimaging has begun to map cerebral mechanisms, while a number of proinflammatory cytokines, a selection of genes and oxidative stress [5] have all been implicated in fatigue pathogenesis. Yet, this work is in its infancy and it will be a number of years until these promising results can translate into a comprehensive etiological model and tangible treatments for those who suffer from fatigue. It is clear that while further research is being conducted to elucidate the biological determinants of fatigue, clinicians must use existing knowledge and tools to support patients now.

In the first instance, it is sensible to confirm that the fatigue being reported is not easily explained by recognized treatment side effects or comorbidities. For example, heightened fatigue may result from analgesics [6], corticosteroids that disrupt hypothalamic-pituitary-adrenal axis functioning, or $\beta$-blockers [7]. It may also be caused by significant anemia or thyroid dysfunction. While the screening of such reversible causes of fatigue is important, ultimately the true origins of fatigue will likely prevail elsewhere for the majority of our patients.

The second stage of fatigue management should focus on adopting and targeting the insights provided by clinical and epidemiological studies. In particular, a number of studies have provided evidence that report reductions in rheumatic fatigue as a secondary benefit to immunosuppressant therapies [8-12]. Now, in order to better improve fatigue in a larger

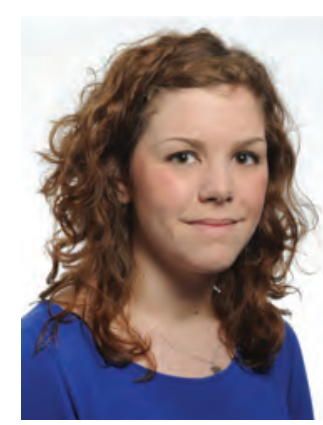

Katie Druce

Author for correspondence:

Epidemiology Group, Institute of Applied Health Sciences, University of Aberdeen, Aberdeen, UK

k.druce@abdn.ac.uk

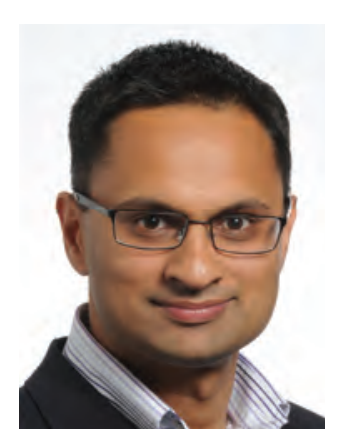

Neil Basu

Epidemiology Group, Institute of Applied Health Sciences, University of Aberdeen, Aberdeen, UK 
proportion of patients, supplementary interventions require exploration. Such interventions will require the targeting of factors that drive fatigue improvements and it is becoming apparent that disease activity is unlikely to be the principal culprit. Anecdotal reports as to the fatigue-disease activity association are not consistently supported by epidemiological studies; lupus patients continue to report substantial fatigue during times of low, or no, disease activity and patients with fibromyalgia experience a high level of fatigue despite a lack of inflammation.

\footnotetext{
"The effects of pain, as well as the effects of disease activity, are mediated by sleep problems, which are a second key mediator of fatigue."
}

As a result, researchers are increasingly turning to the use of mediation models to clarify the proponents of fatigue change and identify possible targets for treatment. So far, these models have demonstrated that fatigue reductions are likely a downstream impact of disease activity on a number of other factors. It is of importance that many of these factors, which are psychosocially influenced, appear to be common between diseases - not only then may the etiology of fatigue be common between diseases, but perhaps treatment could be too. In particular, a strong body of evidence has broadly implicated pain, sleep disruptions, depression and disability as mediators of fatigue and those which should be targeted to optimize treatment of the symptom.

It has long since been established that there is a strong link between fatigue and pain, and it is known that a number of pain-relieving drugs prompt improvements in fatigue [13]. It is therefore unsurprising that the evidence indicates that pain mediates the effect of disease activity and drives improvements in fatigue [14,15]. The effects of pain, as well as the effects of disease activity, are mediated by sleep problems, which are a second key mediator of fatigue $[16,17]$. Yet, while attempts to reduce fatigue via reductions in pain may be fruitful, attempts to do so via reductions in sleep problems are less so; attempts to rest and sleep sufficiently appear not to reduce or manage fatigue [16]. Instead, it seems that improved sleep hygiene should be used to stave off heightened fatigue, rather than to improve an existing fatigue state.

Depressive symptoms also mediate fatigue changes and patient's perception of their condition [6,15]. Although the relationship is complicated by the identification of fatigue as a symptom of depression in addition to overlapping definitions and measurements of the symptoms, it has been shown that both a history of depression and current poor mental health mediates fatigue change [15]. It is therefore likely that future stratification according to the presence of a depressive history may aid the appropriate triage of patients towards more relevant treatment pathways. Lastly, disability, another possible target for treatment, may cause heightened fatigue due to the increased physical effort required to complete daily tasks [18] and indeed changes in disability have been found to be linked to changes in fatigue $[8,19]$.

For those seeking to treat fatigue, it is promising that a number of nonpharmacological intervention studies, which include physical activity and psychosocial interventions, have been shown to reduce the problem [20]. However, fatigue is rarely the primary outcome of these trials which tend to exert their effect by targeting the established mediators of fatigue (disability, pain, sleep and depressive symptoms) in isolation. It is therefore not surprising to observe rather modest effect sizes in terms of fatigue alleviation. That being said, existing interventions represent an ideal foundation for symptom management and it is possible that fatigue focused modifications underpinned by the targeting of multiple mediators will produce enormous gains in management. Such trials also provide the ideal vehicle to better delineate more precise mediators, for example, coping dysfunctions, which in turn will optimize effectiveness even further.

\section{Conclusion \& future perspective}

Fatigue is a common and burdensome symptom across the rheumatic diseases for which there is currently no recommended treatment. Novel and promising work is being conducted to determine the biological mechanisms of fatigue, but it will take many years to turn this into tangible treatments. Patients can no longer remain in the burdensome state of high fatigue and so clinicians must use the data and techniques readily at hand to treat the symptom.

\section{"...it is variables such as pain, depression, sleep and disability which appear to represent ideal targets for treatment."}

The treatment of the underlying disease process offers an important starting block in the management process, but as mediation models indicate, these improvements may not reflect direct improvements in disease activity. Instead, it is variables such as pain, depression, sleep and disability which appear to represent ideal targets for treatment. It should be acknowledged that there is an increasing focus on the potential existence of differing fatigue phenotypes between which the causes and therefore treatments of fatigue may differ. As a result, it may be that we should not only be asking what, but also who to target for treatment. 
However, until the types of fatigue are better understood, treatment should comprise a best-fit approach to provide relief for the most number of patients possible. It is proposed that this will be achieved through the modification and application of additional management programs, which should comprise multidisciplinary nonpharmacological interventions, to be initiated alongside pharmaceutical disease management strategies.

\section{References}

1 Pollard LC, Choy EH, Gonzalez J, Khoshaba B, Scott DL. Fatigue in rheumatoid arthritis reflects pain, not disease activity. Rheumatology 45(7), 885-889 (2006).

2 Hewlett S, Nicklin J, Treharne GJ. Fatigue in musculoskeletal conditions. Reports on the rheumatic diseases (series 6). In: Topical Reviews 1. Keat A, Mawer F (Eds). Arthritis Research Campaign, Chesterfield, UK (2008).

3 Mancuso CA, Paget SA, Charlson ME. Adaptations made by rheumatoid arthritis patients to continue working: a pilot study of workplace challenges and successful adaptations. Arthritis Care Res. 13(2), 89-99 (2000).

4 Repping-Wuts H, Uitterhoeve R, van Riel P, Van Achterberg T. Fatigue as experienced by patients with rheumatoid arthritis (RA): a qualitative study. Int. J. Nurs. Stud. 45(7), 995-1002 (2008).

5 Norheim KB, Jonsson G, Omdal R. Biological mechanisms of chronic fatigue. Rheumatology 50(6), 1009-1018 (2011).

6 Power JD, Badley EM, French MR, Wall AJ, Hawker GA. Fatigue in osteoarthritis: a qualitative study. $B M C$ Musculoskelet. Disord. 9(1), 63 (2008).

7 Papadopoulos AS, Cleare AJ. Hypothalamic-pituitaryadrenal axis dysfunction in chronic fatigue syndrome. Nat. Rev. Endocrinol. 8(1), 22-32 (2012).

8 Druce K, Jones GT, Macfarlane GJ, Basu N. Patients receiving anti-tumour necrosis factor therapies experience clinically important improvements in rheumatoid arthritis related fatigue: results from the British society for rheumatology biologics register. Rheumatology 53(Suppl. 1), i44 (2014).

9 Chauffier K, Salliot C, Berenbaum F, Sellam J. Effect of biotherapies on fatigue in rheumatoid arthritis: a systematic review of the literature and meta-analysis. Rheumatology 51(1), 60-68 (2012).

10 Revicki DA, Luo MP, Wordsworth P, Wong RL, Chen N, Davis JC. Adalimumab reduces pain, fatigue, and stiffness in patients with ankylosing spondylitis: results from the

\section{Financial \& competing interests disclosure}

The authors have no relevant affiliations or financial involvement with any organization or entity with a financial interest in or financial conflict with the subject matter or materials discussed in the manuscript. This includes employment, consultancies, honoraria, stock ownership or options, expert testimony, grants or patents received or pending, or royalties.

No writing assistance was utilized in the production of this manuscript.

adalimumab trial evaluating long-term safety and efficacy for ankylosing spondylitis (ATLAS). J. Rheumatol. 35(7), 1346-1353 (2008).

11 Meijer JM, Meiners PM, Vissink A et al. Effectiveness of rituximab treatment in primary Sjögren's syndrome: a randomized, double-blind, placebo-controlled trial. Arthritis Rheum. 62(4), 960-968 (2010).

12 Thatayatikom A, White AJ. Rituximab: a promising therapy in systemic lupus erythematosus. Autoimmun. Rev. 5(1), 18-24 (2006).

13 Staud R. Peripheral and central mechanisms of fatigue in inflammatory and noninflammatory rheumatic diseases. Curr. Rheumatol. Rep. 14(6), 539-548 (2012).

14 Brophy S, Davies H, Dennis MS et al. Fatigue in ankylosing spondylitis: treatment should focus on pain management. Semin. Arthritis Rheum. 42(4), 361-367 (2013).

15 Druce K, Jones GT, Macfarlane GJ, Basu N. Improvements in rheumatoid arthritis related fatigue are driven by reductions in pain, not disease activity - results from the British Society for Rheumatology Biologics Register for Rheumatoid Arthritis. Arthritis Rheum. (2014) (In Press).

16 McKinley PS, Ouellette SC, Winkel GH. The contributions of disease activity, sleep patterns, and depression to fatigue in systemic lupus erythematosus. Arthritis Rheum. 38(6), 826-834 (1995).

17 Nicassio PM, Moxham EG, Schuman CE, Gevirtz RN. The contribution of pain, reported sleep quality, and depressive symptoms to fatigue in fibromyalgia. Pain 100(3), 271-279 (2002).

18 Stebbings $S$, Treharne GJ. Fatigue in rheumatic disease: an overview. Int. J. Clin. Rheum. 5(4), 487-502 (2010).

19 Rat AC, Pouchot J, Fautrel B, Boumier P, Goupille P, Guillemin F. Factors associated with fatigue in early arthritis: results from a multicenter national French cohort study. Arthritis Care Res. 64(7), 1061-1069 (2012).

20 Cramp F, Hewlett S, Almeida C et al. Non-pharmacological interventions for fatigue in rheumatoid arthritis. Cochrane Database of Syst. Rev. 8, CD008322 (2013). 